EPJ manuscript No.

(will be inserted by the editor)

\title{
Nonequilibrium Phase Transitions into Absorbing States
}

\section{Focused around the pair contact process with diffusion}

\author{
Su-Chan Park ${ }^{1}$ and Hyunggyu Park ${ }^{2}$ \\ 1 Institut für Theoretische Physik, Universität zu Köln, Zülpicher Str. 77, 50937 Köln, Germany, e-mail: psc@thp.uni-koeln.de \\ 2 School of Physics, Korea Institute for Advanced Study, Seoul 130-722, Korea, e-mail: hgpark@kias.re.kr
}

Received: date / Revised version: date

\begin{abstract}
Systems with absorbing (trapped) states may exhibit a nonequilibrium phase transition from a noise-free inactive phase into an ever-lasting active phase. We briefly review the absorbing critical phenomena and universality classes, and discuss over the controversial issues on the pair contact process with diffusion (PCPD). Two different approaches are proposed to clarify its universality issue, which unveil strong evidences that the PCPD belongs to a new universality class other than the directed percolation class.
\end{abstract}

PACS. 64.60.Ht Dynamic critical phenomena - 05.70.Ln Nonequilibrium and irreversible thermodynamics - 89.75.Da Systems obeying scaling laws

\section{Introduction}

The absorbing phase transition (APT) which may occur in ' a system with absorbing states has been an active research topic in nonequilibrium statistical mechanics with possible applications to a wide range of areas in physics, chemistry, biology, and sociology [12/3/4. Microscopic states into which probability is collected are called absorbing. Once a system is trapped into absorbing states, it cannot escape. Absorbing states sometimes form an absorbing space of many states around which a system wanders forever, but does not escape out of the absorbing space. Such an example is the state with only one particle diffusing in physical space for the pair contact process with diffusion (PCPD); see Sect. 3.

In the study of the APT, two different quantities may serve as order parameter 1 . One is the steady state density of the activity (outside of the absorbing states) in the thermodynamic limit ${ }^{2}$, say $\rho$ whose definition varies from system to system, and the other is the survival probability, say $P_{s}$, with which a system does not get trapped into absorbing states forever. Since a finite number of activity

\footnotetext{
1 Although the APT has nothing to do with the orderdisorder transition, the jargon "order parameter" has been used to name the indicator of the phase transition which takes zero in one phase and non zero in the other.

${ }^{2}$ For systems with finite volume of the configurational state space, the presence of the absorbing state always lets a system fall into it eventually [5]. So, as in the equilibrium statistical mechanics, the thermodynamic limit (infinite extension of the configurational state space) is indispensable to study a nontrivial APT.
}

(or local activity) means zero activity density in the thermodynamic limit but non zero survival probability, $\rho=0$ in general does not imply $P_{s}=0$ although the reverse is always true. In a sense, $\rho\left(P_{s}\right)$ is the order parameter for the "macroscopic" ("microscopic" 3 ) APT. Hence, these two quantities might pinpoint different transition points in principle, see Sect. 3 for an example. However, with proper initial conditions and definitions for each order parameter, these transitions points may coincide in general.

Besides the difference mentioned above, nature of the phase transition described by these order parameters can be different even if they locate the same critical point. To clarify this point, consider the branching process with spontaneous death $(A \rightarrow 2 A$ with rate $\sigma$ and $A \rightarrow 0$ with rate $\lambda$ ). The absorbing state of this model is a state without $A$. This problem is the linear one step process in Ref. [5], which can be solved easily and one may find the survival probability as

$$
P_{s}=\left\{\begin{array}{cc}
0 & \text { if } \lambda \geq \sigma \\
\frac{\sigma-\lambda}{\sigma} & \text { if } \lambda \leq \sigma
\end{array} .\right.
$$

\footnotetext{
3 The term "microscopic" is employed because the survival probability has been mainly analyzed in practice when the activity is initially localized in an infinite lattice. In this case, the macroscopic density $\rho$ remains zero for all time by definition though the number of activity may increase indefinitely with finite probability. Hence, the meaningful activity density $\rho$ in the thermodynamic limit should be examined with a finite initial density.
} 
Su-Chan Park, Hyunggyu Park: Nonequilibrium Phase Transitions into Absorbing States

On the other hand, the mean number of particles (activity), say $\langle n\rangle$, behaves as

$$
\frac{\partial\langle n\rangle}{d t}=(\sigma-\lambda)\langle n\rangle \Rightarrow\langle n\rangle=n_{0} e^{(\sigma-\lambda) t},
$$

with the initial value $n_{0}$. Both quantities are singular at $\lambda=\sigma$, but $P_{s}$ increases continuously unlike $\langle n\rangle$ which shows a sharp jump at the transition point. Hence in the study of the APT, $P_{s}$ and $\rho$ should be studied independently and both quantities are important in understanding the APT.

In what follows, however, we restrict ourselves to the study of the "macroscopic" APT, or the scaling behavior of $\rho$ and quantities related to it like the correlation functions. Like equilibrium phase transitions, the APT is characterized and classified by the critical exponents; the order parameter exponent $\beta$, correlation length exponent $\nu_{\perp}$, relaxation time exponent $\nu_{\|}$, and so on, which are defined as

$$
\rho \sim\left(p_{c}-p\right)^{\beta}, \xi \sim\left|p-p_{c}\right|^{-\nu_{\perp}}, \tau \sim\left|p-p_{c}\right|^{-\nu_{\|}},
$$

where $p$ is the external tuning parameter with $p_{c}$ to be the critical point, $\xi$ is the correlation length, and $\tau$ is the relaxation time. One can define other critical exponents but with the aid of the scaling ansatz most of critical exponents can be deduced from the above exponents [6]. For example, the density decays as $t^{-\delta}$ at criticality with $\delta=\beta / \nu_{\|}[\underline{6}$.

To categorize universality classes according to the critical exponents and to understand what properties combine different systems into the same universality class are the main goals in this field. Some understanding has emerged from the numerical and analytical studies. Section 2 briefly summarizes the well-established universality classes, such as the directed percolation (DP), the directed Ising (DI), the parity conserving (PC) classes and so forth. The last 10 years have witnessed intensive discussion and hot debate on a simple but very elusive interacting particle system, the PCPD. In Sect. 3, we will critically review on the issue regarding its universality class. To settle the controversy, two different approaches have been proposed by the authors, which is the subject of Sect. 4. We draw a conclusion in Sect. 5 .

\section{Universality classes}

The simplest non-trivial model which shows an APT might be the contact process $(\mathrm{CP})$ which is an interacting hard core particle system on a $d$ dimensional lattice with the creation of a particle by a neighboring particle and the spontaneous annihilation $(A \rightarrow 2 A, A \rightarrow 0)$ 7. The particle vacuum state is the only absorbing state in which the system cannot escape by the prescribed rules. Hence the order parameter is the density of occupied sites (or particles). The $\mathrm{CP}$ shares the critical behavior with the directed percolation (DP) the preferred direction of which is interpreted as the time direction of the $\mathrm{CP}$ and the open channel of which as a particle 2]. The DP has the rapidityreversal (or time-reversal) symmetry [6]8] which associates the microscopic APT with the macroscopic one, that is, which renders $P_{s}$ to scale equivalently to $\rho$. A nice illustration of the connection in the context of the bond directed percolation can be found in Ref. [2]; see also Ref. [4].

After extensive numerical studies regarding the universality class of the APT, it has been conjectured 4 that the APT model with a single absorbing state should belong to the DP class if symmetry or conservation is not involved 89]10. The robustness of the DP class extends to the systems with infinitely many absorbing states like the pair contact process (PCP) [11, at least in its stationary property. In the PCP, the creation and annihilation of particles are only mediated by two particles which form a nearest neighbor pair on a lattice $(2 A \rightarrow 3 A$, $2 A \rightarrow 0)$. So any configuration devoid of a pair is an absorbing state and the number of absorbing states increases exponentially with system size. Clearly, the order parameter should be the pair density not the particle density (auxiliary field density) which is nonzero at stationarity irrespective of the phase. The robustness of the DP class suggests that the absence of symmetry or conservation of the order parameter should render the system to belong to the DP class, irrespective of the existence of the auxiliary field associated with infinitely many absorbing states. Although the stationary property of the PCP conforms with the DP scaling, there is still vivid discussion regarding the dynamic scaling of its spreading [12 13/14]15], which is beyond the scope of this paper.

Other universality classes have been found by adding symmetry or conservation in dynamics. The directed Ising (DI) class involves the $Z_{2}$ (Ising) symmetry in dynamics, the evolution operator of which is invariant under the $Z_{2}$ symmetry operation [16]17/18]. Naturally, the typical DI systems include two equivalent absorbing states. The DI scaling also applies to systems with two equivalent groups of multiple absorbing states 192021. The conservation of the particle number of modulo 2 reveals another universality class called as the parity conserving (PC) class 22 23, which coincides with the DI class in one dimension. In higher dimensions, both classes can be described by the trivial mean-field theory. Recently another universality class (generalized voter class) has been examined, which also coincide with the DI class in one dimension 24]. It is worthy to note that the DI (and PC) class returns to the DP class immediately with the introduction of a symmetry breaking field or a conservation breaking dynamics $25|26| 27 / 28$.

There had been an attempt to find a new universality class by studying models with higher symmetry than $Z_{2}$ or $\bmod (q)$ conservation with $q>2$. However, all models so far show a trivial critical behavior even in one dimension, in that the absorbing phase is found to be always unstable against the dynamics increasing activity of the order parameter.

All the systems explained up to now can be described by the single component order parameter. Richer behavior

\footnotetext{
4 This statement is termed as the "DP conjecture".
} 
is anticipated when multi species are involved. For example, the interaction of the order parameter with a conserved field triggers a different type of universal behavior depending on the activity of the conserved field 2930. In fact, any system involving multi-particle reactions can be interpreted as a multi-species particle system. One can map the PCP to the multi-species model by identifying a pair as a particle of one kind and a single isolated particle as a particle of another kind. More general cases will be discussed in subsequent sections.

\section{Pair contact process with diffusion}

The pair contact process with diffusion (PCPD) is an extended model of the PCP with hopping of particles allowed for. To be specific, the dynamics of the PCPD in $d$ dimensions consists of hopping, pair annihilation, and creation by a pair, which is symbolically summarized as

$$
\begin{aligned}
& \left.\begin{array}{l}
A \emptyset \rightarrow \emptyset A \\
\emptyset A \rightarrow A \emptyset
\end{array}\right\} \text { with rate } \frac{D}{d}, \\
& A A \rightarrow \emptyset \emptyset \text { with rate } \frac{p}{d}, \\
& \left.\begin{array}{l}
A A \emptyset \\
\emptyset A A
\end{array}\right\} \rightarrow A A A \text { with rate } \frac{1-p}{2 d}
\end{aligned}
$$

where $A(\emptyset)$ stands for an occupied (a vacant) site on a lattice and $0 \leq p \leq 1$. The PCP corresponds to the above rules with $D=0$ which make any configuration without two particles in a row (a pair) absorbing. Due to the diffusion, however, a state without a pair but many isolated particles is not absorbing any longer. Only both the particle vacuum and the state with only one particle in the whole system are absorbing. Since the particle density of both absorbing states of the PCPD is zero in the thermodynamic limit, it can play the role of the order parameter in contrast to the PCP case. Needless to say, the pair density may also serve as an order parameter.

Due to the lack of a process to eliminate a single particle (no single particle reaction) without particle collisions, the conventional survival probability and the density might locate different transition points. To elucidate, consider the PCPD in higher dimensions than 2. If initially two particles are located somewhere in an infinite lattice (just outside of the absorbing space), the survival probability is always finite because of the nonrecurrence of the random walk even for the case of $p=1$. That is, the survival probability predicts the absence of the "microscopic" absorbing phase. However, the macroscopic critical point should be located at finite $p$. The reason is as follows: The mean density $\rho$ of particles in $d$ dimensions satisfies the (exact) equation,

$$
\frac{d \rho}{d t}=(1-3 p) \rho_{p}-(1-p) \rho_{t}
$$

where $\rho_{p}\left(\rho_{t}\right)$ means the pair (triplet) density. If $p>\frac{1}{3}$, the steady state value of the pair and triplet density should be zero, which is clear by Eq. (5). If $\rho$ were not zero in the steady state, macroscopic number of pairs should be formed by the diffusion, which is contradictory to the observation that $\rho_{p}=0$. Hence $\rho$ should approach to zero if $p>\frac{1}{3}$ and the critical point should be not larger than $\frac{1}{3}$ and the "macroscopic" absorbing phase is present in any dimension 5 . In the above argument, we assume the existence of the steady state even in the thermodynamic limit.

As the above consideration reveals, the single-particle diffusion plays a crucial role in changing the nature of the conventional microscopic APT, which has nothing to do with the DP for higher dimensions than 2. However, this does not resolve the controversy regarding the universality class of the PCPD. First, the difference of the microscopic APT does not guarantee that of the macroscopic one. A good example is the PCP whose macroscopic APT is characterized by the DP scaling but whose microscopic APT is known to be nonuniversal 12 13 14 15. Second, the main issue is not any dimensional PCPD but one dimensional PCPD where it is not fully clear that the microscopic APT is equivalent to the macroscopic APT 31/32.

In fact, the difference between the PCPD and the DP is well appreciated for two or more dimensional systems. First consider the mean field equation. The mean field equation for the PCPD can be found by replacing $\rho_{p}$ and $\rho_{t}$ with $\rho^{2}$ and $\rho^{3}$, respectively in Eq. (5) which reads

$$
\frac{\partial \rho}{\partial t}=(1-3 p) \rho^{2}-(1-p) \rho^{3}
$$

The mean field critical exponents are $\beta=1$ and $\delta=$ $\beta / \nu_{\|}=\frac{1}{2}$ 33134, which are different from those of DP $\left(\beta=\nu_{\|}=1\right)$ 2]. Since the upper critical dimension of the PCPD is believed to be 2 [35], for most physically relevant cases $(d \geq 2)$ the PCPD does not belong to the DP class.

For the one dimensional PCPD, however, the numerically estimated critical exponents of the PCPD are so similar to those of the DP that the possibility for the PCPD to belong to the DP class has been raised [36 37]. Interestingly, the critical exponent 6 which describes the density decay with time at criticality has floated from $\simeq 0.28[38$ to less than 0.185 [37. with time, which is due to the strong corrections to scaling. For comparison, the numerical value of $\delta_{\mathrm{DP}}$ is $\simeq 0.15946$ [39].

An argument in favor of the DP scenario was suggested by Hinrichsen [37. The starting point of the argument is the numerical observation that the dynamic exponent $z$ is smaller than 2 which is the dynamic exponent of the random walk. Hence, if coarse graining is performed according to the PCPD dynamic exponent, the diffusing isolated particles will stop moving asymptotically and the

\footnotetext{
${ }^{5}$ To resolve the difference between the microscopic and macroscopic APT, a new definition of survival of the system has been suggested: The system without a pair is considered as (temporarily) absorbing. With this definition, it has been shown that two order parameters exhibit the APT's at the same transition point at least in one dimension 31/32.

${ }^{6}$ From now on, by critical indices without subscript are always meant those of the PCPD.
} 
long time behavior of the PCPD should be same as that of the PCP which belongs to the DP.

We would like to make some comments as to this argument. To begin, it is not at all clear why the dynamics of the wandering isolated particles is decoupled asymptotically from that of the active clusters. If not, the dynamic scaling of the isolated particles should be affected by the complex environmental geometry of active clusters. Therefore there is no ground for the belief that the diffusion of isolated particles remains governed by the random walk dynamic exponent $z_{\mathrm{RW}}=2$. If the system scales in one way as a whole (not decoupled), the coarse graining argument does not lead to the zero diffusion constant of the isolated particles. Second, the Hinrichsen's argument set the upper bound for the dynamic exponent $z \leq 2$ in order to be self-consistent. Based on this, one can comment on the two dimensional PCPD which seems definitely not in the DP class. This leaves us only one option to take $z=2$ for the two (and higher) dimensional PCPD even without any logarithmic correction. However, the upper critical dimension of the PCPD is believed to be 2 , where the logarithmic correction is expected and the numerical results seem to support its presence [35. Therefore, even if one may accept the decoupling of two different fields in the PCPD, his argument seems not working at two dimensions.

Regardless of the universality issue, one can ask why the PCPD has such strong corrections to scaling which are the main obstacle in numerical study. The long term memory effect was suggested as a possible origin of the strong corrections to scaling though it is not clearly answered how the finite mean life time from the life time distribution $P(\tau) \sim \tau^{-2.2}$ can trigger such strong corrections to scaling or even a new scaling deviated from the DP 31. Only a possible scenario was contemplated that the interacting theory may force the system to flow into a non-DP fixed point even with finite mean life time, reflecting on the similar result found in the Lévy flight DP systems [4041. Due to the fact that the memoryless case of the generalized PCPD clearly shows the DP scaling [31, it is certain that the memory effect plays an important role in determining the universality class of the PCPD. Another reason of believing the role of the memory comes from the model tp12 (for the definition, see below) and similar varieties of models with rules $n A \rightarrow(n+m) A$ and $l A \rightarrow(l-k) A$ with $n>l$ and $m, k>0$ which are numerically shown to belong to the DP [42]. Same as the PCPD, the tp12 $(n=3, l=2, m=1, k=2)$ with zero diffusion constant has infinitely many absorbing states and belongs to the DP class [43. In contrast to the PCPD ( $n=2, l=2, m=1, k=2)$, the diffusion turns out not to affect the universality class of the tp12. In Ref. [43, it is argued that the effective lack of the memory in the tp12, which is clearly seen from the space-time configuration Fig. 1, renders any model with $n>l$ to belong to the DP class.

Another complicated feature of the PCPD has arisen in the analytical study. The formulation of the field theory based on the single field turned out impossible and it

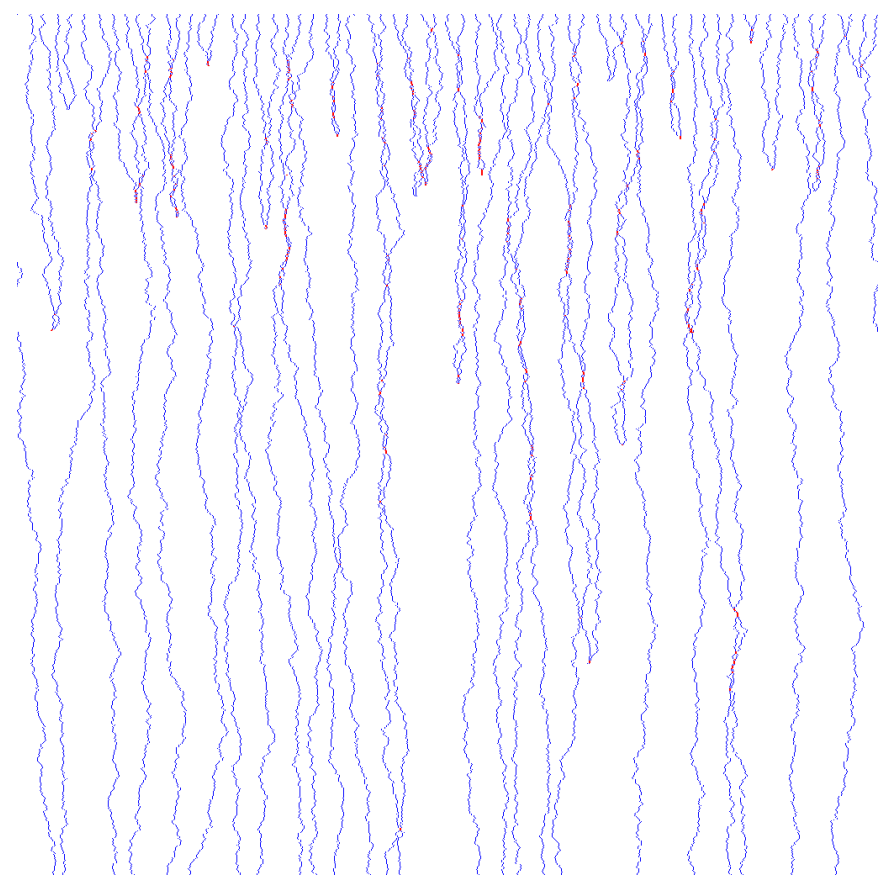

Fig. 1. (Color online) Space-time configuration of the tp12 at criticality with a sparse initial condition. The time flows from top to bottom and the cross section by a horizontal line is the space configuration at specified time. The periodic boundary conditions are employed. Effective lack of the memory (see the text) means the seemingly absence of the branching process $3 A \rightarrow 4 A$ due to the low probability of forming a triple before a pair is annihilated.

is argued that the nonperturbative treatment still cannot cure this failure [44. The lesson from the study is that at least two independent field should be included in the proper action to understand the PCPD in the field theoretical framework. The proper field theory which is local in space and time, if exists, should take two independent fields into account. In principle, one can write down the Langevin equation equivalent to the PCPD using two independent fields [45, but the proper analytical tool does not seem to be at hand.

\section{How to tackle the problem}

The one dimensional PCPD seems very difficult, if not impossible, to tackle directly. In the numerical front, the strong corrections to scaling prohibits researchers from measuring the critical exponents accurately 8 . In the analytical front, the failure of the field theory by the single field requires an ingenious treatment of the problem.

In this section, we will try to convince readers that the PCPD does not belong to the DP class. First, introducing

7 The effective field theory might be described by a single field with nonlocal interaction, which in principle can be achieved by integrating out some of the fields in a local action.

8 The most recent numerical studies still do not provide a conclusive evidence 46 47. 


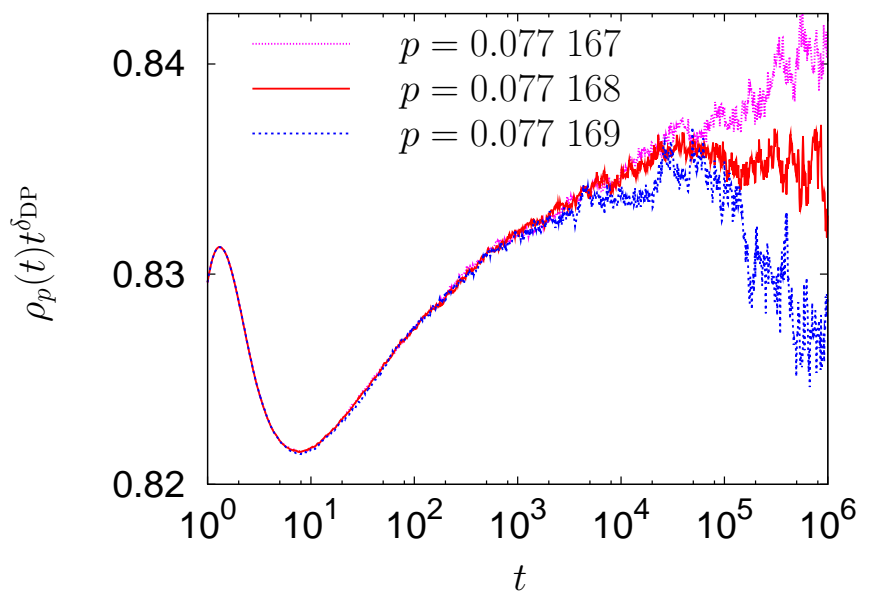

Fig. 2. (Color online) Semi-logarithmic plot of the pair density multiplied by $t^{\delta_{\mathrm{DP}}}$ with $\delta_{\mathrm{DP}}=0.1594$ vs time for the PCP with biased branching. In the active (absorbing) phase, the curve veers up (down) and at criticality the flat straight line is expected, which renders the estimation of the critical point as $p_{c}=0.077168(1)$ with error in the last digit.

the bias, we will argue that the failure of the field theory by the single field is also generic even in one dimension. Second, studying the crossover model from the PCPD to the DP by introducing the single particle dynamics, the difference of these two classes will be clarified.

\subsection{Effect of Biased Diffusion}

As discussed at the end of Sect. 3, the PCPD is supposed to be described by two independent "elementary excitations", that is, the isolated particle-field and the pair-field.

Even if we find the proper field theory for the PCPD, it might not resolve the controversy on the one dimensional PCPD. For example, what if the fixed point of the PCPD turns out not to be reachable by the perturbation expansion to the one dimensional system just as that of the branching annihilating walk (BAW) with even number of offspring [4]? However, before worrying about the above scenario, we must take a first step to answer the most elementary question whether it is absolutely necessary to employ two independent fields in the one dimensional PCPD, in contrast to the Reggeon field theory of the DP which needs a single field. The answer may not resolve the controversy definitely, but should be regarded as a big step in understanding the difference between the PCPD and the DP class.

Recently, the present authors have suggested to check if the one dimensional PCPD should have two independent relevant fields via adding a bias in diffusion [49:34. We got some hints from the study of the two species annihilation model $A+B \rightarrow \emptyset$ which shows different decaying behavior in the presence of the relative bias between $A$ and $B$ from that without bias 5051.

Since the DP has only one relevant field, the bias should be gauged away by the Galilean transformation. Besides, lots of complicated models which belong to the DP class also turn out to be robust against the bias 49433. Implementing the relative bias between relevant fields and observing how the system responds can be a litmus test to check numerically whether the single field is enough to describe the system. In this context, we introduced the driven pair contact process with diffusion (DPCPD) and studied it [49:34].

Before delving into the DPCPD, we should emphasize that the presence of two "independent" fields is not sufficient to observe the change of critical behavior by the bias. Although the PCP is described by two independent fields, we expect that the branching bias which triggers the relative bias between pairs and isolated particles does not alter the critical behavior because the PCP belongs to the DP class. In Fig. 2, we showed how the pair density behaves around the critical point of the PCP with biased branching. As in the PCP, $p$ is the pair annihilation probability and $1-p$ is the creation probability. The branching bias is given such that a created particle is always placed at the right neighbor of the pair once that site is vacant. Figure 2 locates the critical point $p_{c}$ as $p_{c}=0.077$ 168(1) by exploiting the power law behavior of the pair density, say $\rho_{p}$, at criticality. As can be seen, the critical decay exponent still takes the DP value $\left(\delta_{\mathrm{DP}}\right)$, which implies that the relative bias is irrelevant in the PCP.

Now let us continue the discussion on the DPCPD. In Ref. [49, the DPCPD shows clear distinction from both the DP and the PCPD scaling in one dimension, which was confirmed again by the cluster approximation along with the coherent anomaly method analysis 34 . This result is not expected if there is only one relevant field in the PCPD. Hence the field theory of the PCPD should be different from the Reggeon field theory even in one dimension, which provides a strong evidence of a non-DP scaling in the PCPD.

Another interesting feature of the DPCPD is the dimensional reduction. The one dimensional DPCPD is numerically found to have the same critical behavior as the two dimensional PCPD 49. The dimensional reduction by the biased diffusion is actually reported in different areas in nonequilibrium statistical physics. One example is the two species annihilation model, $A+B \rightarrow 0$. When there is (no) relative bias between $A$ and $B$, the density decays as $t^{-(d+1) / 4}\left(t^{-d / 4}\right)$ when $d<3(d<4)$ and as $t^{-1}$ when $d>3(d>4) 50$ 51. Another example can be found in the study of the self organized criticality. It is rigorously proved that the upper critical dimension of the directed sand pile model is 3 unlike its undirected version whose upper critical dimension is 4 [52].

\subsection{Learning from crossover scaling}

Another evidence that the PCPD does not belong to the DP was provided by the study of the crossover from the PCPD to the DP [53]. Along with the dynamics in Eqs. (4), we introduce the single particle annihilation/creation

$$
\left.A \stackrel{w q}{\longrightarrow} 0, \quad \begin{array}{l}
A \emptyset \\
\emptyset A
\end{array}\right\} \stackrel{w(1-q) / 2}{\longrightarrow} A A
$$


where $0 \leq q \leq 1$. If $w \neq 0$, the system shows the DP scaling behavior $[53$.

What can we expect about the scaling behavior near the PCPD critical point for finite $w$ if PCPD does belong to the DP class? From the rigorous study about the "crossover" based on the stochastic equivalence shown in [54, the "crossover" between the same universality classes is expected to have two characteristics. First, the phase boundary is expected to meet the PCPD point linearly. Second, the critical amplitudes which is defined as $\rho(t) t^{\delta_{\mathrm{DP}}}$ for cases with finite but small $w$ should collapse with the critical amplitude of the PCPD at $w=0$. Our recent numerical results show a nontrivial crossover exponent from the PCPD to the DP and the phase boundary approaches the PCPD point in a singular (nonlinear) way [53. This provides another strong evidence that the PCPD is different from the DP.

In fact, one should be cautioned in interpreting the singular behavior of the phase boundary. The linearity of the phase boundary may become complicated by the nontrivial singularity arising between the PCP and the DP crossover [43, which corresponds to the dynamics modeled by Eqs. (4) and (7) with $D=0$. However, this singularity has nothing to do with the universality class. In a sense, the model with $w=0$, i. e., the PCP, is pathological in that the configuration volume occupied by the absorbing states is macroscopic, which is not the case for finite $u[\sqrt{10}$. Hence there is an inherent singularity, actually discontinuity of the particle (auxiliary field) density, close to $w=0$, which is reflected by the nontrivial crossover exponent although the PCP belongs to the DP class [43]. If we introduce $3 A \rightarrow 0$ rather than Eq. (77), we reproduced two characteristics of the "crossover" between models in the same universality class; see Fig. 3. The results summarized in Fig. [3] are rather easily conceivable because the operator corresponding to $3 A \rightarrow 0$ is irrelevant in the RG sense and moreover there is no singularity of the auxiliary field density near $w=0$. In this context, the single particle branching/annihilation introduced to the PCP is relevant because this operator changes the structure of the absorbing configurations, which is manifest by the singularity at $w=0$ [3], though it does not change the universality class.

Since the volume of the absorbing states in the configuration space for the PCPD is zero in the thermodynamic limit, there is no singularity arising from the auxiliary field near the crossover from the PCPD to the DP. Hence the reason of the singularity in the PCPD-DP crossover should be understood in another context. The easiest answer may be that the PCPD does not belong to the DP. Of course, there could be a hidden unknown reason for the PCPD to lead to a singular crossover behavior and its possibility cannot be fully excluded. Hence we studied a

\footnotetext{
9 We use the quotation mark to mention the "crossover" between models in the same class because the "crossover" is not truly a crossover in convention.

10 When $w$ is finite, the only absorbing state is the particle vacuum.
}

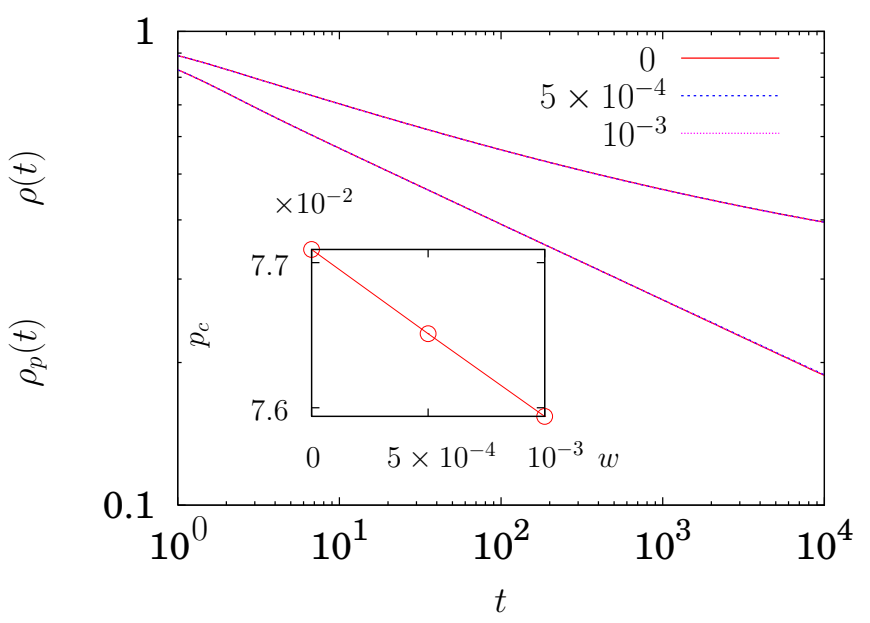

Fig. 3. (Color online) Log-log plots of the particle density (upper curves) and the pair density (lower curves) at criticality vs $t$ for the PCP "crossover" model with the dynamics $3 A \rightarrow 0$ which occurs with probability $w$. The critical points are $0.0770905(5), 0.07651(1)$, and 0.07594(1) for $w=0$, $w=5 \times 10^{-4}$, and $w=10^{-3}$, respectively. The difference among cases with $w=0$ and finite $w$ is hardly seen. Inset: The phase boundary near $w=0$. The line meets the vertical axis linearly.

similar model to the PCPD whose non diffusing counter part has infinitely many absorbing states and which is known to belong to the DP class. It is found that the crossover model based on the tp12 have two properties of the "crossover" among models belonging to the same universality class 43 . We are unable to conceive of a mechanism that would explain these observations, while maintaining the PCPD and tp12 in the same universality class; a more natural interpretation is that the two models belong to different classes, so that the PCPD does not fall in the DP class. All together, our study of the crossover scaling strongly suggests that the PCPD does not belong to the DP class.

\section{Conclusion}

Up to now, we discussed about the hotly debated issue of the pair contact process with diffusion (PCPD). Among many scenarios proposed at the first stage of the controversy [33, only two seem to have survived; does the PCPD belong to the directed percolation class or form a different universality class from any other known one? In this paper, we gave evidences in favor of the second scenario.

At first, the fact that the biased diffusion drastically changes the critical behavior of the PCPD suggests that the one dimensional PCPD should be described by the two independent relevant "elementary excitations" which is not the case of the Reggeon field theory (DP). Second, the nontrivial crossover scaling arising between the PCPD and the DP strongly suggests that the PCPD should not belong to the DP class. 
Although the main interest of this paper is the PCPD, the methods employed to clarify the universality issue of the PCPD (the role of the biased diffusion and the crossover) are generally applicable to many other systems, some of which are currently under investigation.

\section{References}

1. J. Marro, R. Dickman, Nonequilibrium Phase Transitions in Lattice Models (Cambridge University Press, Cambridge, 1999)

2. H. Hinrichsen, Adv. Phys. 49, (2000) 815

3. G. Ódor, Rev. Mod. Phys. 76, (2004) 663

4. S. Lübeck, Int. J. Mod. Phys. B 18, (2004) 3977

5. N.G. van Kampen, Stochastic processes in physics and chemistry, 2nd edn. (North Holland, Amsterdam, 1997)

6. P. Grassberger, A. de la Torre, Ann. Phys. (NY) 122, (1979) 373

7. T.E. Harris, Ann. Prob. 2, (1974) 969

8. H.-K. Janssen, Z. Phys. B: Condens. Matter 42, (1981) 151

9. P. Grassberger, Z. Phys. B: Condens. Matter 47, (1982) 365

10. G. Grinstein, Z.-W. Lai, D.A. Browne, Phys. Rev. A 40, (1989) 4820

11. I. Jensen, Phys. Rev. Lett. 70, (1993) 1465

12. M.A. Muñoz, G. Grinstein, R. Dickman, J. Stat. Phys. 91, (1998) 541

13. A. Jiménez-Dalmaroni, H. Hinrichsen, Phys. Rev. E 68, (2003) 036103

14. R. Dickman, Phys. Rev. E 53, (1996) 2223

15. P. Grassberger, H. Chaté, G. Rousseau, Phys. Rev. E 55, (1997) 2488

16. M.H. Kim, H. Park, Phys. Rev. Lett. 73, (1994) 2579

17. H. Park, M.H. Kim, H. Park, Phys. Rev. E 52, (1995) 5664

18. W. Hwang, S. Kwon, H. Park, H. Park, Phys. Rev. E 57, (1998) 6438

19. W. Hwang, H. Park, Phys. Rev. E 59, (1999) 4683

20. M.C. Marques, J.F.F. Mendes, Eur. Phys. J. B 12, (1999) 123

21. H.S. Park, H. Park, J. Korean Phys. Soc. 38, (2001) 494

22. P. Grassberger, J. Phys. A: Math. Gen. 22, (1989) L1103

23. H. Takayasu, A.Y. Tretyakov, Phys. Rev. Lett. 68, (1992) 3060

24. O.A. Hammal, H. Chaté, I. Dornic, M.A. Muñoz, Phys. Rev. Lett. 94, (2005) 230601

25. H. Park, H. Park, Physica A 221, (1995) 97

26. S. Kwon, H. Park, Phys. Rev. E 52, (1995) 5955

27. K.E. Bassler, D.A. Browne, Phys. Rev. Lett. 77, (1996) 4094

28. H. Hinrichsen, Phys. Rev. E 55, (1997) 219

29. R. Kree, B. Schaub, B. Schmittmann, Phys. Rev. A 39, (1989) 2214

30. F. van Wijland, K. Oerding, H.J. Hilhorst, Physica A 251, (1998) 179

31. J.D. Noh, H. Park, Phys. Rev. E 69, (2004) 016122

32. R. Dickman and M.A.F. de Menezes, Phys. Rev. E 66, (2004) 045101(R)

33. M. Henkel, H. Hinrichsen, J. Phys. A: Math. Gen. 37, (2004) R117

34. S.-C. Park, H. Park, Phys. Rev. E 71, (2005) 016137
35. G. Ódor, M.C. Marques, M.A. Santos, Phys. Rev. E 65, (2002) 056113

36. G.T. Barkema, E. Carlon, Phys. Rev. E 68, (2003) 036113

37. H. Hinrichsen, Physica A 361, (2006) 457

38. E. Carlon, M. Henkel, U. Schollwöck, Phys. Rev. E 63, (2001) 036101

39. I. Jensen, J. Phys. A: Math. Gen. 29, (1996) 7013

40. H.-K. Janssens, K. Oerding, F. van Wijland, H.J. Hilhorst, Eur. Phys. J. B 7, (1999) 137

41. H. Hinrichsen, M. Howard, Eur. Phys. J. B 7, (1999) 635

42. J. Kockelkoren, H. Chaté, Phys. Rev. Lett. 90, (2003) 125701

43. S.-C. Park, H. Park, Phys. Rev. E (in press); e-print arxiv/0706.2973

44. H.-K. Janssens, F. van Wijland, O. Deloubriere, U.C. Täuber, Phys. Rev. E 70, (2004) 056114

45. S.-C. Park, Eur. Phys. J. B 50, (2006) 327

46. M.M. de Oliveira and R. Dickman, Phys. Rev. E 74, (2006) 011124

47. S. Kwon and Y. Kim, Phys. Rev. E 75, (2007) 042103

48. J. Cardy, U.C. Täuber, Phys. Rev. Lett. 77, (1996) 4780

49. S.-C. Park, H. Park, Phys. Rev. Lett. 94, (2005) 065701

50. K. Kang, S. Redner, Phys. Rev. Lett. 52, (1984) 955

51. K. Kang, S. Redner, Phys. Rev. A 32, (1985) 435

52. D. Dhar, R. Ramaswamy, Phys. Rev. Lett. 63, (1989) 1659

53. S.-C. Park, H. Park, Phys. Rev. E 73, (2006) 025105

54. S.-C. Park, J.-M. Park, Phys. Rev. E 71, (2005) 026113 